\title{
A new release from astrocytes
}

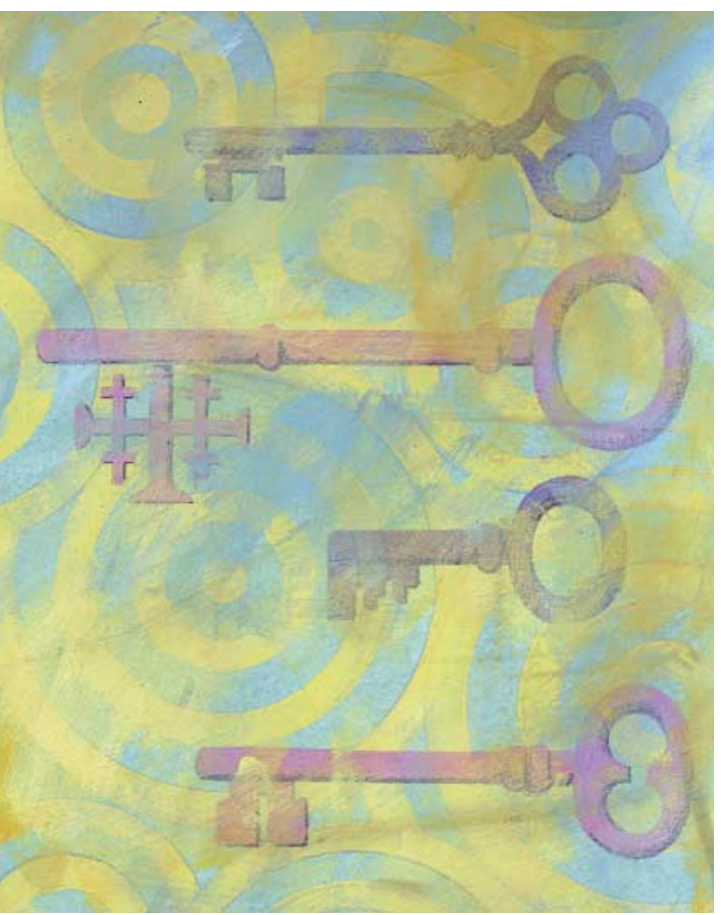

In recent years, astrocytes have emerged as key modulators of neuronal excitability and synaptic transmission. It is known that they can use the chemical transmitter glutamate to 'talk' to neurons and synapses, but the nature of the glutamate storage and release machinery has been unclear. Now, however, Bezzi and colleagues present evidence that astrocytes release glutamate through a SNARE-dependent exocytic mechanism, which is strikingly similar to the mechanism of neurotransmitter release at nerve terminals.

Bezzi et al. showed that astrocytes in the rat hippocampus express transcripts that code for the vesicular glutamate transporters VGLUT1 and VGLUT2, which are responsible for glutamate uptake into synaptic vesicles. Using immunogold cytochemistry and electron microscopy, the authors pinpointed the location of these molecules to vesicular organelles, which were observed in regions of astrocytic processes that impinged on neuronal structures. In some cases, astrocytic vesicles faced neuronal NMDA ( $N$-methyl-D-aspartate) receptors at

nearly synaptic distance. The vesicles also expressed cellubrevin - a member of the vesicular SNARE (v-SNARE) family of proteins, which regulate the fusion of exocytic vesicles with the plasma membrane

Next, the authors used cultured astrocytes to examine whether the vesicles underwent regulated exocytosis in response to group I metabotropic glutamate receptor (mGluR) activation. They observed the behaviour of the vesicles using total internal reflection fluorescence imaging, a technique that allows dynamic cellular processes to be visualized at high resolution. They labelled the cells with acridine orange, a dye that accumulates in acidic cellular compartments in a self-quenched state and produces a flash of fluorescence when it is released at the cell surface. The astrocytes were also engineered to express VGLUT1 or VGLUT2 tagged with enhanced green fluorescent protein. The authors showed that the mGluR agonist dihydroxyphenylglycine evoked flashes of fluorescence at the cell membrane, concomitant with the disappearance of some of the VGLUT-expressing vesicles.

So, there was clear evidence that exocytosis was taking place in response to mGluR activation, but were the astrocytes actually releasing glutamate? To find out, Bezzi et al. co-cultured astrocytes with 'glutamate-sniffing'

\section{The highs and lows of synaptic plasticity}

\author{
Although the importance of NMDA \\ (N-methyl-D-aspartate) receptors for long- \\ term plasticity at many synapses is well \\ established, it is unclear how activation of \\ these receptors can lead to either potentiation \\ or depression of synaptic activity under \\ different circumstances. Now, Liu and \\ colleagues suggest that the subunit \\ composition of the receptors might be the \\ determining factor in the direction of \\ plasticity. \\ At synapses in hippocampal area CA1, \\ specific patterns of activation can lead to \\ long-term potentiation (LTP) or long-term \\ depression (LTD), both of which depend on \\ the activation of NMDA receptors. High- \\ frequency stimulation produces LTP, whereas \\ low-frequency stimulation leads to LTD. \\ Partially blocking NMDA synapses with \\ an antagonist, APV (D,L-2-amino- \\ 5-phosphophonovaleric acid), blocks the \\ induction of LTP but not that of LTD, and this \\ finding led to the idea that the direction of
}

plasticity depends on the degree of activation of the NMDA receptors and therefore the level and kinetics of NMDA receptor-gated $\mathrm{Ca}^{2+}$ influx. But Liu et al. have come up with a different explanation.

NMDA receptors consist of NR1 and NR2 subunits. There are four types of NR2 subunit, with the primary ones in the CA1 region of the hippocampus being NR2A and NR2B. When the authors used an NR2B-specific antagonist, such as ifenprodil, they were unable to induce LTD, even though LTP was unaffected.
Conversely, an NR2A-specific blocker, NVPAAM077, prevented the induction of LTP but had little effect on LTD. These results support a new model, in which differential activation of NMDA receptors containing either the NR2A or the NR2B subunit controls whether synaptic activity is potentiated or depressed.

If this is the case, a low dose of APV that only partially blocked NMDA receptor activation would be expected to prevent LTP but not LTD, because NR2A-containing receptors are more sensitive to APV than

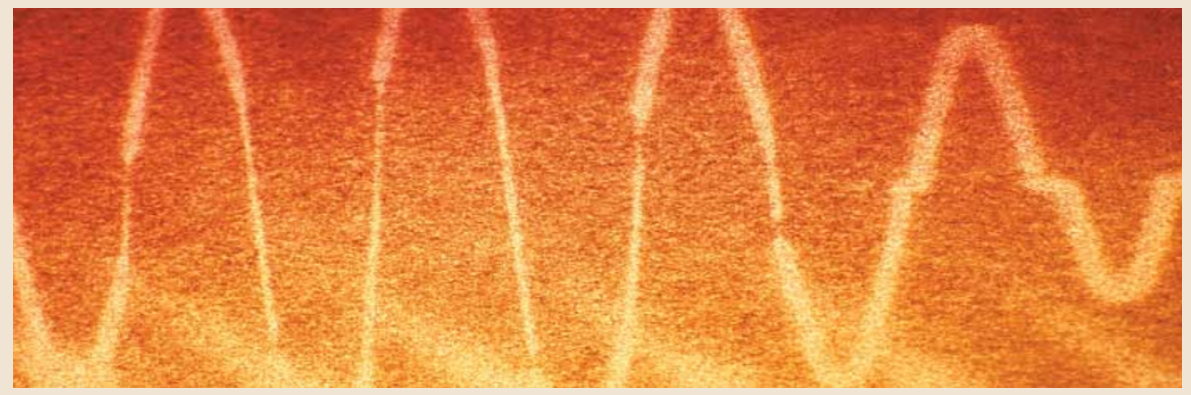


cells - insulinoma-1 (INS-1) cells that respond to glutamate application with a rapid elevation in their intracellular calcium concentration owing to the expression of NMDA receptors. The authors showed that the flashes of fluorescence at the astrocyte membrane were followed by an NMDA receptor-dependent increase in intracellular calcium in the INS-1 cells, confirming that glutamate was being released through regulated exocytosis.

Although the astrocytic glutamate-releasing vesicles are similar to synaptic vesicles in many ways, there are also some intriguing differences. For example, the groups of vesicles were often smaller and less well-organized than the vesicles at presynaptic terminals. Also, they express the v-SNARE cellubrevin, whereas synaptic vesicles mostly express VAMP2 (vesicle-associated membrane protein 2). In the future, it will be interesting to explore the implications of these similarities and differences for neuron-glia communication.

Heather Wood

\section{(2) References and links}

original RESEARCH PAPER Bezzi, P. et al. Astrocytes contain a vesicular compartment that is competent for regulated exocytosis of glutamate. Nature Neurosci. 7, 613-620 (2004)

FURTHER READING Haydon, P. G. Glia: listening and talking to the synapse. Nature Rev. Neurosci. 2, 185-193 (2001) |

Newman, E. A. New roles for astrocytes: regulation of synaptic transmission. Trends Neurosci. 26, 536-542 (2003)

those that contain NR2B. The idea is also consistent with previous findings, such as the fact that the NR2A/NR2B ratio increases during development, and LTD is more difficult to induce in more mature animals. However, there are also some problems for the theory. For example, a previous study by Tang et al. found that overexpression of NR2B subunits in the forebrain of mice increased levels of LTP, not LTD.

Furthermore, different rules might apply in different parts of the brain.

Further work will no doubt help to clarify such issues, and should establish how different stimulation protocols might preferentially activate receptors that contain different types of subunit. It should also investigate what might happen downstream of receptor activation: there is already evidence that NR2A-containing receptors might couple to different signalling pathways from NR2Bcontaining receptors, and this provides a potential mechanism for their different effects on long-term plasticity.

(9)) References and links Rachel Jones ORIGINAL RESEARCH PAPER Liu, L. et al. Role of NMDA receptor subtypes in governing the direction of hippocampal synaptic plasticity. Science 304, 1021-1024 (2004) FURTHER READING Tang, Y. P. et al. Genetic enhancement of learning and memory in mice. Nature 401, 63-69 (1999)

\section{ION CHANNELS}

\section{Crystal-clear interactions}

Calcium channels are the latest family to give in to the power of crystallography and reveal their structural details. Three recent papers have disclosed the atomic structure of the interacting domains between $\alpha$ - and $\beta$-subunits of voltagegated calcium channels, challenging some previous ideas about the workings of the channel.

Voltage-gated calcium channels comprise different subunits - the $\alpha_{1}$-subunit forms the pore of the channel, and the $\alpha_{2}-, \beta$ - and $\gamma$-subunits modulate channel function. The $\beta$-subunit, in particular, has a profound effect on several channel properties, including activation and inactivation rates and surface expression. Previous studies had narrowed down the $\alpha_{1}$ - and $\beta$-regions that mediate their interaction — the so-called $\alpha_{1}$-interaction domain (AID) and a conserved core of the $\beta$-subunit, which includes what was regarded as the $\beta$-interaction domain (BID). The three papers report on the crystal structure of the conserved core of several $\beta$-subunits on their own and bound to the AID.

Perhaps the most surprising result from the three papers is that the BID does not really interact with the $\alpha$-subunit. Instead, it is largely buried within the $\beta$-core and is more relevant for the structural integrity of the actual binding site, which, as it turns out, is structurally reminiscent of another protein family - membraneassociated guanylate kinases (MAGUKs).

MAGUKs are known to function as scaffolds at the synapse, a function for which their several PDZ domains are critical. The PDZ domains, which are absent in the core of the $\beta$-subunit, precede an $\mathrm{SH} 3$ domain and a guanylate kinase domain. A linker domain joins these two regions and, together, these three domains constitute the region of homology between MAGUKs and the conserved $\beta$-core. The structural analysis showed that the domain of the $\beta$-subunit that binds the AID is a hydrophobic cleft within the region homologous to the guanylate kinase domain of MAGUKs; the BID, by contrast, lies in the linker domain.

From the functional perspective, the structural results provide good clues about the way in which the $\beta$-subunit can affect channel inactivation. The interaction between the two subunits places the $\beta$-region near the intracellular end of a pore-lining segment of the $\alpha$-subunit that is important for switching the channel off, raising the possibility that the $\beta$-subunit can affect the movement of this segment through electrostatic or physical interactions.

Last, the AID seems to occupy a small part of the region of homology between MAGUKs and the $\beta$-subunit. As this region contains other protein-protein interaction modules, such as the $\mathrm{SH} 3$ domain, it is conceivable that additional molecules bind to this region, potentially increasing the complexity of calcium channel modulation.

Juan Carlos López, Chief Editor, Nature Medicine

\section{(2) References and links}

ORIGINAL RESEARCH PAPERS Van Petegem, F. et al. Structure of a complex between a voltage-gated calcium channel $\beta$-subunit and an $\alpha$-subunit domain. Nature 429, 671-675 (2004) | Opatowsky, Y. et al. Structural analysis of the voltage-dependent calcium channel $\beta$ subunit functional core and its complex with the $\alpha_{1}$ interaction domain. Neuron 42, 387-399 (2004) | Chen, Y. et al. Structural basis of the $\alpha-\beta$ subunit interaction of voltage-gated $\mathrm{Ca}^{2+}$ channels. Nature 429, 675-680 (2004) 